\title{
One-dimensional dynamic simulations of slip complexity of earthquake faults
}

\author{
Jeen-Hwa Wang and Ruey-Der Hwang \\ Institute of Earth Sciences, Academia Sinica, P.O. Box 1-55, Nankang, Taipei, Taiwan
}

(Received October 18, 1999; Revised November 13, 2000; Accepted November 13, 2000)

\begin{abstract}
Slip complexity of earthquake faults is studied based on an N-degree-of-freedom dynamical spring-slider system in the presence of slip-law-type, velocity- and state-dependent friction. Simulation results based on such a friction law show that slip complexity depends on the inhomogeneous distribution of the breaking strengths (including its pattern and degree) along the fault and nonlinear velocity- and state-dependent friction. However, for the given model parameters the former is more important than the latter in controlling slip complexity. Frictional effects obviously appear only when the distribution of the breaking strengths is inhomogeneous. In addition, the stiffness ratio, defined as the ratio of the coil spring strength, $K_{c}$, to the leaf spring strength, $K_{l}$, is also a factor in controlling slip complexity.
\end{abstract}

\section{Introduction}

Slip complexity has long been observed from both the field survey and from the inversion of earthquake source ruptures (cf. Scholz, 1990; Kanamori, 1994). A complex slip distribution has been interpreted in terms of two phenomenological models. One of them is specified with barriers (areas where no slip occurs during a mainshock) proposed by Das and Aki (1977) and the other with asperities (areas where large slip takes place during a mainshock) suggested by Kanamori and Stewart (1978). However, the physical nature of asperities and barriers is not well known (cf. Kanamori, 1994). Hence, it is significant to explore the causes to yield slip complexity of earthquake faults.

Numerous authors also theoretically studied this problem. Carlson and her co-authors (Carlson, 1991; Carlson and Langer, 1989; Carlson et al., 1991) stated that selforganization of repeated ruptures of uniform faults gives rise to slip complexity when inertial effects and nonlinear velocity-weakening friction are included. They stressed the importance of non-linearity of friction on slip complexity. On the other hand, Rice (1993) strongly argued that all of simulation results from the self-organizing models have been sensitive to the spatial discretization used. He stressed the importance of material heterogeneities and defined a coherent slip patch size, $h^{*}$. So-called self-organizing model faults, having a numerical cell size $h>h^{*}$, are inherently discrete, thus resulting in Gutenberg-Richter-type complexity for small events. Based on a model through the discretization from a continuum one, Shaw (1994) stated that slip complexity is mainly caused by non-linearity of velocity- and statedependent friction rather than by the matter that the grid size $h$ is larger than a critical length $h^{*}$ of the model. Knopoff (1996) considered both heterogeneous breaking strengths and non-linearity of friction to be two important factors in

Copy right (C) The Society of Geomagnetism and Earth, Planetary and Space Sciences (SGEPSS); The Seismological Society of Japan; The Volcanological Society of Japan; The Geodetic Society of Japan; The Japanese Society for Planetary Sciences. influencing slip complexity.

In this work, we first study the effects due to two factors: the inhomogeneous distribution of the breaking strengths and non-linearity of velocity- and state-dependent friction. Secondly, from simulation results we explore which is the major factor in controlling slip complexity of earthquake faults. In this study, we use an N-degree-of-freedom spring-slider model, proposed by Burridge and Knopoff (1967), in the presence of velocity- and state-dependent friction, to approximate earthquake dynamics.

\section{Theory}

The N-degree-of-freedom dynamic spring-slider model (see Fig. 1) consists of $\mathrm{N}$ sliders of equal mass, $m$, and springs with one slider being linked by a coil spring of strength, $K_{c}$, with the other. A slider is also pulled by a leaf spring of strength, $K_{l}$, on a moving plate with a constant velocity, $V_{p}$. At time $t=0$, all the sliders rest in the individual equilibrium states. The $i$-th slider $(i=1, \ldots, N)$ is located at position $x_{i}$, measured from its initial equilibrium position, along the $x$ axis. A slider is subjected to a velocity- and state-dependent frictional force, $F_{i}\left(\theta_{i}, v_{i}\right)$, where $\theta_{i}$ and $v_{i}\left(=d x_{i} / d t\right)$ are the state variable and the sliding velocity, respectively, of the $i$-th slider. The equation of motion of the $i$-th slider is

$$
\begin{aligned}
m\left(d^{2} x_{i} / d t^{2}\right)= & K_{c}\left(x_{i+1}-2 x_{i}+x_{i-1}\right) \\
& -K_{l}\left(x_{i}-V_{p} t\right)-F_{o}\left(\theta_{i}, v_{i}\right) .
\end{aligned}
$$

In Eq. (1), $F_{i}\left(\theta_{i}, v_{i}\right)$ equals to $\mu_{i} \sigma_{n} A$, where $\mu_{i}$ is the friction strength, $\sigma_{n}$ is the normal pressure and $A$ is the contact area between a slider and the moving plate.

Experimental and theoretical studies show that two effects, i.e., a direct effect and an evolution one, affect the dynamic friction strength (cf. Dieterich, 1979; Ruina, 1983; Marone, 1998). The direct effect shows an instantaneous change of the friction strength with a change in velocity, while the evolution effect evolves with slip following a change in velocity and is proportional to the negative log of sliding velocity. Un- 


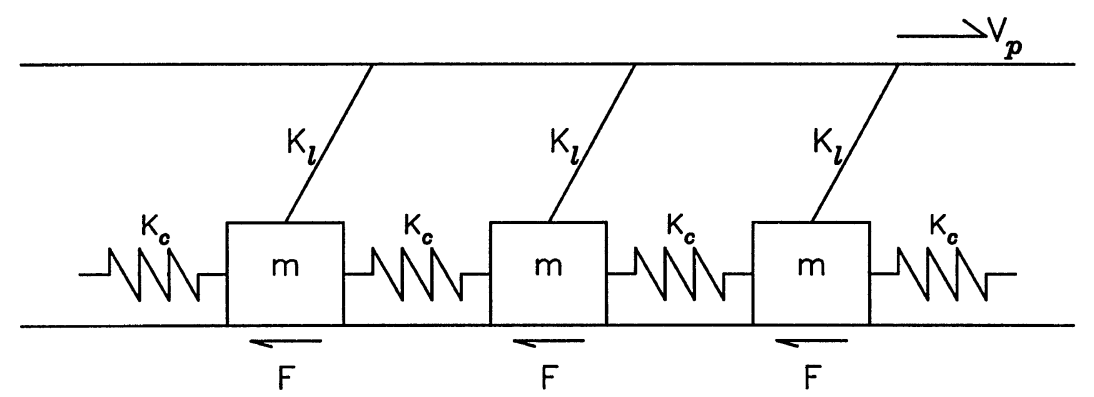

Fig. 1. An N-degree-of-freedom dynamical spring-slider system.

stable slip in rock can result only when the evolution effect is larger than the direct one. The one-state-variable, velocityand state-dependent friction strength, $\mu$, which employs a state variable, $\theta$, defined by Ruina (1983), has a form of $\mu=\mu_{o}+a \ln \left(v / v_{o}\right)+b \ln \left(\theta v_{o} / D_{c}\right)$, where $\mu_{o}$ is the breaking strength (or the static friction strength), $v$ is the sliding velocity, $v_{o}$ is a constant reference velocity, and $D_{c}$ is the characteristic slip distance. This leads to $\mu=\mu_{o}$ when $v=v_{o}$ and $\theta=D_{c} / v_{o}$. Contributions to the total friction strength is scaled by $a$ for the direct effect and by $b$ for the evolution one. Two evolution laws are proposed to control the state variable (cf. Marone, 1998). One of them is the slip law: $d \theta / d t=-\left(\theta v / D_{c}\right) \ln \left(\theta v / D_{c}\right)$. When $v=0$, $d \theta / d t$ approximates to zero. This implicates that no evolution occurs when $v=0$. The other is the slowness law: $d \theta / d t=1-\theta v / D_{c}$. When $d \theta / d t=0$, state is proportional to slowness, i.e., $\theta=D_{c} / v$. For the two laws, the steady-state friction strength, $\mu^{s s}$, is $\mu_{o}+(a-b) \ln \left(v / v_{o}\right)$ when $d \theta / d t=0$. This leads to $\mu^{s s}=\mu_{o}$ for both laws when $v=v_{o}$. From the simulation results based on a onedegree-of-freedom spring-slider model, Wang (1999) stated that the slowness law strongly resists the slider to move when the driving velocity is less than $10^{-3} \mathrm{~m} / \mathrm{s}$. Thus, it is not appropriate to apply the slowness law to simulate dynamic earthquake ruptures. Hence, in this study only the slip law is taken into account.

The velocity- and state-dependent dynamic friction strength at the $i$-th slider is represented by $\mu_{i}=\mu_{o i}+$ $a_{i} \ln \left(v_{i} / v_{i o}\right)+b_{i} \ln \left(\theta_{i} v_{i o} / D_{i c}\right)$. The breaking strength, $\mu_{o i}$, is a function of position, with a maximum value of $\mu_{o \text { max }}$. In order to simplify numerical computations, the values of the parameters $a_{i}, b_{i}, v_{o i}$, and $D_{o i}$ are individually considered to be constant at all sliders, i.e., letting $a_{i}=a, b_{i}=b$, $v_{i o}=v_{o}$ and $D_{i c}=D_{c}$. This leads to $F_{i}\left(\theta_{i}, v_{i}\right)=\sigma_{n} A\left[\mu_{o}+\right.$ $\left.a \ln \left(v_{i} / v_{o}\right)+b \ln \left(\theta_{i} v_{o} / D_{c}\right)\right]=F_{o}\left[\gamma_{o i}+\alpha \ln \left(v_{i} / v_{o}\right)+\right.$ $\left.\beta \ln \left(\theta_{i} v_{o} / D_{c}\right)\right]$, where $F_{o}=\mu_{o \text { max }} \sigma_{n} A, \gamma_{o i}=\mu_{o i} / \mu_{o \max }$, $\alpha=a / \mu_{o \max }$, and $\beta=b / \mu_{o \max }$. The slip law becomes $d \theta_{i} / d t=-\left(\theta_{i} v_{i} / D_{c}\right) \ln \left(\theta_{i} v_{i} / D_{c}\right)$. Replacing $F_{i}\left(\theta_{i}, v_{i}\right)$ into Eq. (1), we have

$$
\begin{gathered}
m\left(d^{2} x_{i} / d t^{2}\right)=K_{c}\left(x_{i+1}-2 x_{i}+x_{i-1}\right)-K_{l}\left(x_{i}-V_{p} t\right) \\
-F_{o}\left[\gamma_{o i}+\alpha \ln \left(v_{i} / v_{o}\right)\right. \\
\left.+\beta \ln \left(\theta_{i} v_{o} / D_{c}\right)\right] .
\end{gathered}
$$

To simply deal with the problem, Eq. (2) is normalized in advance. We define three characteristic parameters, i.e.,
$s=K_{c} / K_{l}, \omega_{o}=\left(K_{l} / m\right)^{1 / 2}$, and $D_{o}=F_{o} / K_{l}$. Wang (1995) called the quantity $s$ the stiffness ratio. The quantity $\omega_{o} / 2 \pi$ is the frequency of oscillation of a single slider attached to a leaf spring in the absence of friction. $D_{o}$ is the characteristic displacement of a slider exerted by a force $F_{o}$ through a spring with strength of $K_{l}$. Longer $F_{o}$ yields longer $D_{o}$ when $K_{l}$ is fixed. Obviously, $D_{o}$ and $\omega_{o}$ can be considered to be two significant units to scale the spatial coordinates, $x_{i}$, and time, $t$, respectively. Let $X_{i}=x_{i} / D_{o}, \tau=\omega_{o} t$, and $\Delta=$ $D_{c} / D_{o}$. This leads to $d x_{i} / d t=\left[F_{o} /\left(m K_{l}\right)^{1 / 2}\right] \partial X_{i} / \partial \tau$, $d^{2} x_{i} / d t^{2}=\left(F_{o} / m\right) d^{2} X_{i} / d \tau^{2}$, and $V_{i}=d X_{i} / d \tau$. Hence, the normalized (dimensionless) quantities are $v_{i}=V_{i} / D_{o} \omega_{o}$, $\phi_{i}=\omega_{o} \theta_{i}, v_{p}=V_{p} / D_{o} \omega_{o}$, and $v_{o}=V_{o} / D_{o} \omega_{o} . D_{o} / V_{p}$ is the loading time for a leaf spring to stretch enough for overcoming the breaking strength, and $v_{p}$, is equivalent to the ratio of the slipping time $\omega_{o}^{-1}$ to the loading time.

Based on the above-mentioned quantities, Eq. (2) is normalized to the following form:

$$
\begin{aligned}
d^{2} X_{i} / d \tau^{2}= & s\left(X_{i+1}-2 X_{i}+X_{i-1}\right)-\left(X_{i}-v_{p} \tau\right) \\
& -\left[\gamma_{o i}+\alpha \ln \left(v_{i} / v_{o}\right)+\beta \ln \left(\phi_{i} v_{o} / \Delta\right)\right] .
\end{aligned}
$$

The normalized form of the slip law is $d \phi_{i} / d \tau=$ $-\left(\phi_{i} v_{i} / \Delta\right) \ln \left(\phi_{i} v_{i} / \Delta\right)$. It is obvious that from the friction law, the value of $\mu$ cannot be defined at $v_{i}=0$. However, for dynamic simulations, we cannot discard the case at $v_{i}=0$. Hence, the values of $\phi_{i}$ are all set to be $\Delta / v_{o}$ when $v_{i}=0$ This can lead to $\mu=\mu_{o}$ and avoid the appearance of infinity when $v_{i}=0$. The main parameters controlling the motion of a slider are $s$ (the stiffness ratio), $\alpha$ (the parameter for the direct effect), $\beta$ (the parameter for the evolution effect), $v_{p}$ (the dimensionless velocity of the moving plate), and $\Delta$ (the dimensionless characteristic slip displacement). According to the definition of friction law, the reference velocity $v_{o}$ is not usually regarded as a parameter affecting the friction strength. However, Wang (1999) stressed that $v_{o}$ must be one of the significant parameters controlling the dynamic friction strength. This can be seen in the followings. In addition, the boundary condition at each end slider should be a factor in influencing simulation results. In the following numerical simulations, the two end sliders are fixed and do not move at all.

To understand the variation of friction strength, $\mu$, with sliding velocity, we calculate the variation of $\mu$ with $v$, which increases, in a sine-function form, from 0 to $v_{\max }\left(=10^{-3}\right.$ $\mathrm{m} / \mathrm{s}$ ), through the fourth-order Runge-Kutta method (cf. Press et al., 1986). The values of model parameters $\mu_{o}, a$, and $b$ 


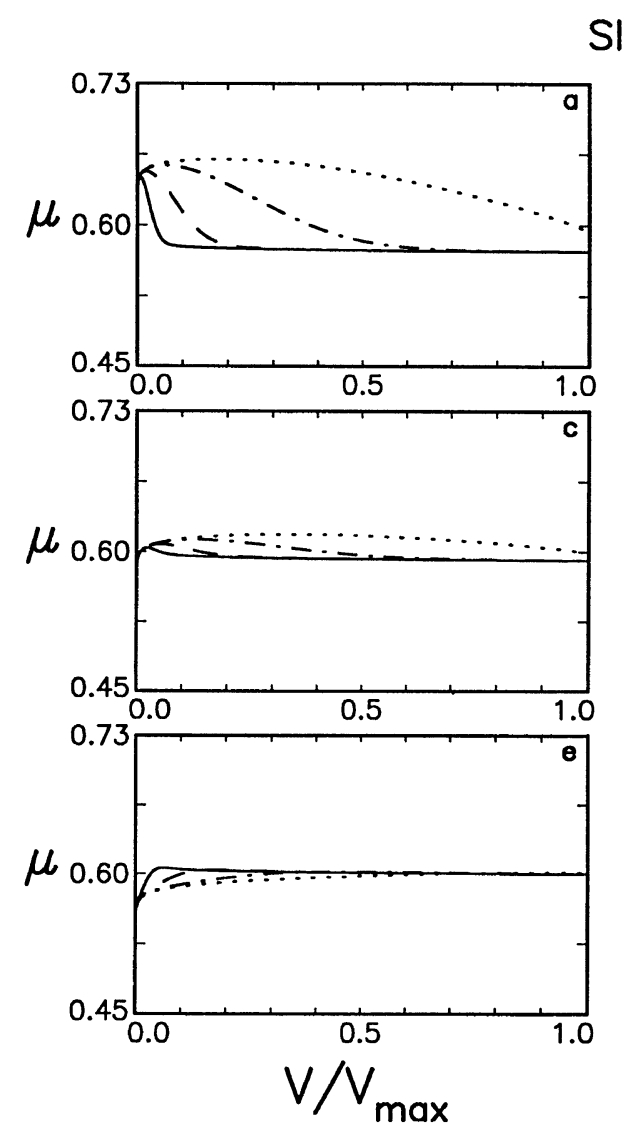

\section{Slip Law}
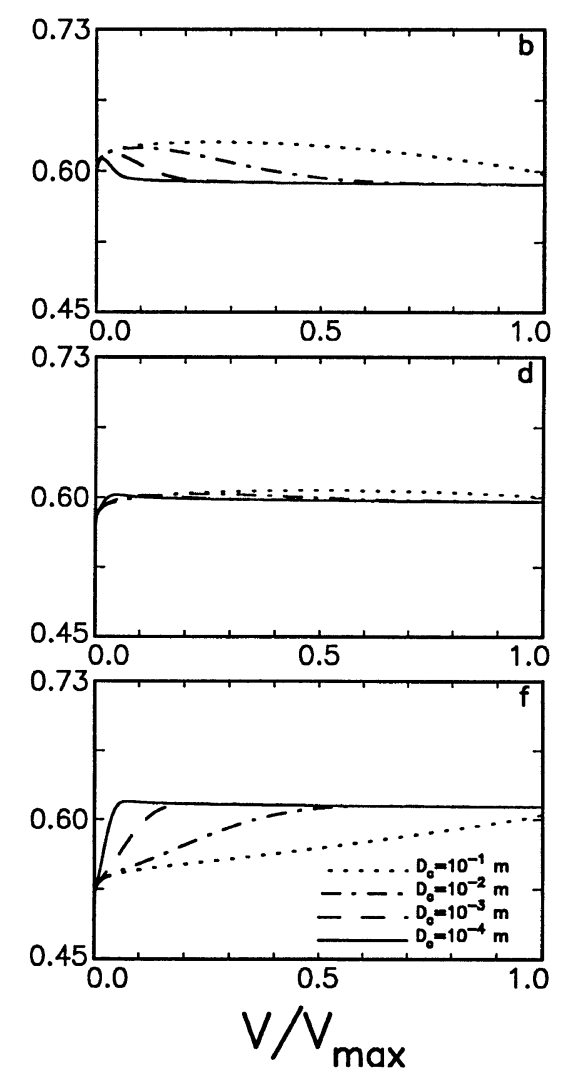

Fig. 2. The variations of the friction strength with normalized sliding velocity, $v / v_{\max }$ (with $v_{\max }=10^{-3} \mathrm{~m} / \mathrm{s}$ ), under the controlling of the slip-type friction law for six values of $v_{o}$ : (a) for $10^{-9} \mathrm{~m} / \mathrm{s}$, (b) for $10^{-6} \mathrm{~m} / \mathrm{s}$, (c) for $10^{-5} \mathrm{~m} / \mathrm{s}$, (d) for $10^{-4} \mathrm{~m} / \mathrm{s}$, (e) for $10^{-3} \mathrm{~m} / \mathrm{s}$, and (f) for $1 \mathrm{~m} / \mathrm{s}$, when $a=6 \times 10^{-3}$ and $b=9 \times 10^{-3}$. For each $v_{o}$, four values of $D_{c}: 10^{-5} \mathrm{~m}$ denoted by a solid line, $10^{-4} \mathrm{~m}$ by a dashed line, $10^{-3} \mathrm{~m}$ by a dashed-dotted-dashed line, and $10^{-2} \mathrm{~m}$ by a dotted line, are considered.

are set to be $0.6,10^{-3}$ and $1.5 \times 10^{-3}$, respectively. For all cases, the initial value of $\theta$ for numerical computations is $D_{c} / v_{o}$. The variations of $\mu$ with $v / v_{\max }$ for six values of $v_{o}$, i.e., $10^{-9} \mathrm{~m} / \mathrm{s}, 10^{-6} \mathrm{~m} / \mathrm{s}, 10^{-5} \mathrm{~m} / \mathrm{s}, 10^{-4} \mathrm{~m} / \mathrm{s}, 10^{-3} \mathrm{~m} / \mathrm{s}$, and $1 \mathrm{~m} / \mathrm{s}$, are shown in Fig. 2. For each $v_{o}$, four values of $D_{c}$, i.e., $10^{-4} \mathrm{~m}, 10^{-3} \mathrm{~m}, 10^{-2} \mathrm{~m}$, and $10^{-1} \mathrm{~m}$, are considered. In each diagram, the solid line, dashed line, dashed-dotted-dashed line, and dotted line represent the results for $D_{c}=10^{-5} \mathrm{~m}$, $10^{-4} \mathrm{~m}, 10^{-3} \mathrm{~m}$, and $10^{-2} \mathrm{~m}$, respectively.

There are two patterns for variations of $\mu$ with $v / v_{\max }$, depending upon $v_{o}$. The value of $v_{o}=10^{-5} \mathrm{~m} / \mathrm{s}$ is a critical one to separate the two patterns. When $v_{o}<10^{-5} \mathrm{~m} / \mathrm{s}$, the value of $\mu$ increases rapidly first from the initial value to a peak value, and then decreases. The velocity range, within which the friction strength increases from the initial value to the peak one, increases with $D_{c}$ and slightly with $v_{o}$. This indicates that the friction force makes resistance to prohibit an object for sliding. The amount of resistance increases with $D_{c}$ and slightly with $v_{o}$ when $v_{o}<10^{-5} \mathrm{~m} / \mathrm{s}$. On the other hand, when $v_{o} \geqq 10^{-5} \mathrm{~m} / \mathrm{s}$, the value of $\mu$ first drops to a value less than 0.6 , then increases from that to a peak value, and finally decreases again. The decrease in the dynamic friction strength at low velocities behaves like an impulse, which supplies additional energy to an object. After the friction strength drops, the dynamic friction strength increases with velocity. The increasing rate of the dynamic friction strength with velocity decreases with increasing $D_{c}$. A rapid increase in the dynamic friction strength can make a stronger resistance to an object for sliding than a slow one. Hence, large $v_{o}$ and large $D_{c}$ are beneficial to an object for sliding when $v_{o} \geqq 10^{-5} \mathrm{~m} / \mathrm{s}$.

Earthquake fault zones are usually quite complex, and, thus, the distribution of the breaking strengths is not homogeneous. Inhomogeneous friction strengths might be caused either by a non-uniform distribution of crustal materials or by variable pore pressures (cf. Rice, 1992). Wang (1995) considered a fractal function specified with a fractal dimension, $D$, to describe the inhomogeneous distribution of the breaking strengths. A fractal distribution of the breaking strengths is also considered in this study. The Fourier filtering method developed by Saupe (1988) is applied to yield a fractal distribution with $2^{n}$ ( $n$ is an integer) discrete points. Let $\gamma_{o \text { max }}$ and $\gamma_{o \text { min }}$ be, respectively, the maximum and minimum values of the breaking strengths, and define $R=\left(\gamma_{o \text { max }}-\gamma_{o \text { min }}\right) / \gamma_{o \text { max }}$ to represent the degree of the inhomogeneous distribution of the breaking strengths.

\section{Simulation Results}

In order to explore slip complexity of earthquake faults, we here study the spatial distribution of fault slip through numerical simulations based on Eq. (3) with slip-law-type velocity- and state-dependent friction. The friction strength 

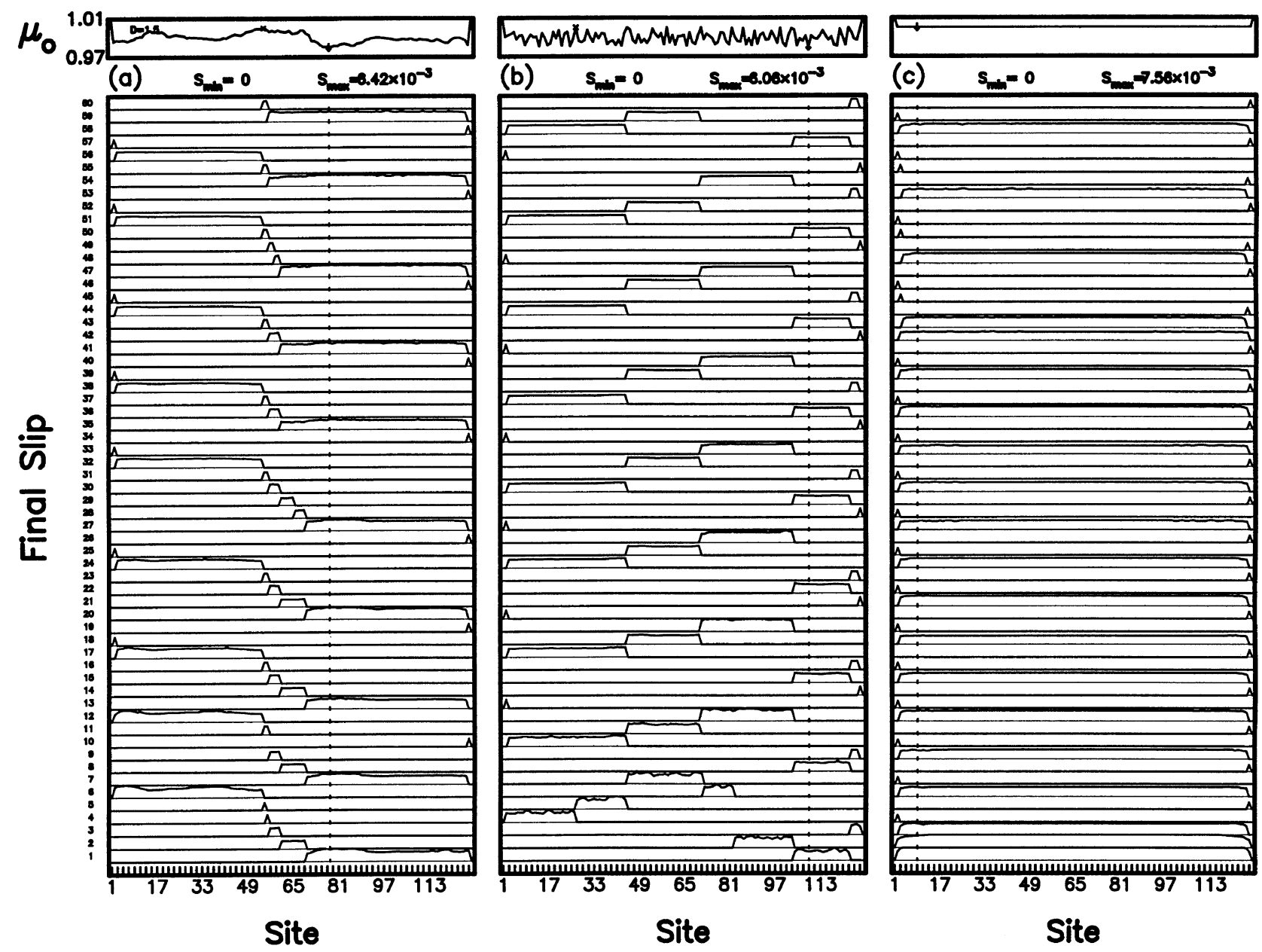

Fig. 3. The spatial distributions of final slip of sixty simulation events for three distributions of the breaking strengths: (a) for a fractal distribution with $D=1.5$, (b) for a random distribution, and (c) for a uniform distribution, when $s=4, \gamma_{o \text { min }}=0.98, \alpha=10^{-2}, \beta=1.5 \times 10^{-2}, v_{o}=1$, and $\Delta=10^{-3}$. The site with $\gamma_{o \max }(=1)$ is denoted by a cross and that with $\gamma_{o}$ min shown by an arrow sign. The dipping line segments at the two end sliders demonstrate the fixed boundary condition. The dotted line in the lower diagrams represents the site with $\gamma_{o \text { min }}$.

is defined only for the positive velocities. This means that no backward motions in the fault are allowed. The simulation procedure is briefly described here. First, we integrate Eq. (3) with friction forward in time. Secondly, we scan all sliders again to find the sliders for which the forces are closest to the individual breaking strengths. The velocity $\nu_{i}(\tau+\delta \tau)$ and the displacement $X_{i}(\tau+\delta \tau)$ of the $i$-th slider at time $\tau+\delta \tau$ is calculated from the acceleration $a_{i}(\tau)\left(=d^{2} X_{i} / d \tau^{2}\right)$ at time $\tau$, i.e., $v_{i}(\tau+\delta \tau)=a_{i}(\tau) \delta \tau$ and $=a_{i}(\tau)(\delta \tau)^{2} / 2$. The value of $\phi_{i}(\tau)$ is computed from the slip-type evolution law through the fourth-order Runge-Kutta method. The computation processes are conducted until all sliders have come to rest. Thirdly, after all sliders come to rest, we add again a loading force from the moving plate to each slider, and then repeat the first and second processes.

The boundary condition must be a factor in affecting simulation results. Since in this study we are only focusing the slip distribution of the model events, the two ends of the model fault do not break in the seismic cycles. Hence, the breaking strengths at two end sliders are set to be infinity for avoiding numerical computations. In other words, we consider the fixed boundary condition. To show the fixed boundary condition, two dipping line segments are plotted at the two end sliders.

For simplification, the inertial effect is considered to be constant for all cases, thus letting $m=1 \mathrm{~kg}$. The strength of the leaf spring, $K_{l}$, is set to be $1 \mathrm{nt} / \mathrm{m}$, thus leading to $\omega_{o}=$ $1 \mathrm{~Hz}$. Four values of $s\left(=K_{c} / K_{l}\right)$, i.e., $2,3,4$, and 5 , are used. It is noted that in this study, since only the normalized equations are used, and only $\omega_{o}$ and $s$ are taken into account, the absolute units of $m, K_{c}$, and $K_{l}$ are less important. For simplification, $D_{o}$ is set to be $1 \mathrm{~m}$ for all cases. Generally, the value of $V_{p}$ is generally about $10^{-9} \mathrm{~m} / \mathrm{s}$, and, thus, the value of $v_{p}$ is $10^{-9}$ when $D_{o} \omega_{o}=1 \mathrm{~m} / \mathrm{sec}$. Since Eq. (3) is a normalized equation, $\gamma_{o \text { max }}$ is 1 for all cases in this study. The difference $a-b$ or $\alpha-\beta$ depends upon the gouge layer thickness and the shear displacement (cf. Marone, 1998). For a bare fault plane, the value of $a-b$ ranges from -0.004 to 0 . The value changes when a gouge layer exists. It changes from a negative value to a positive one when the gouge layer thickness is increased. The value of $a-b$ also decreases with increasing shear displacement. Since the parameter $\alpha$ (or $a$ ) only changes the amount of the direct effect, its value is set to be a constant $\left(=10^{-2}\right)$ in this study. Two values of the parameter $\beta$, i.e., $0.5 \times 10^{-2}$ and $2 \times 10^{-2}$, are considered. The dimensionless reference velocity, $v_{o}$, and 

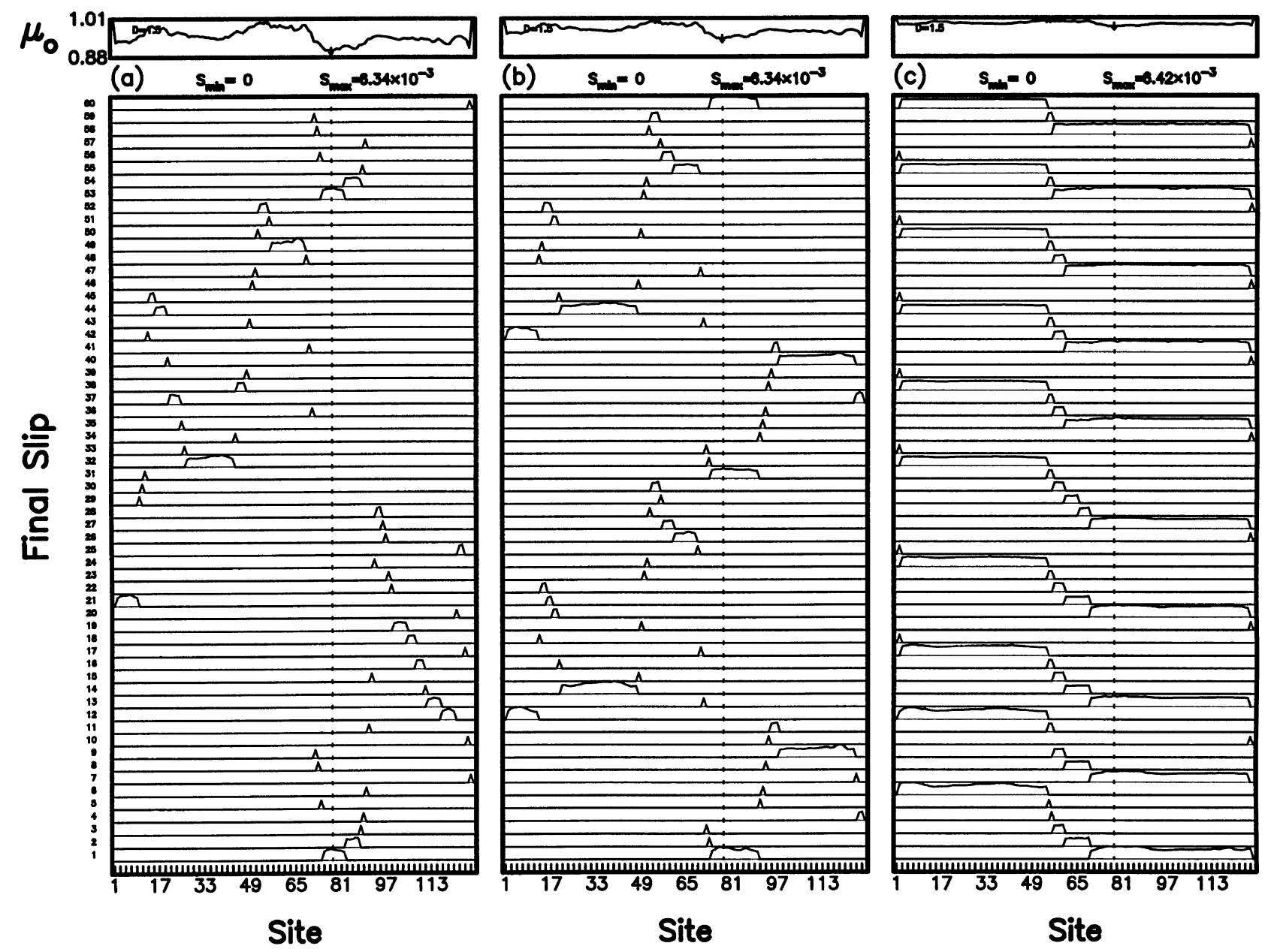

Fig. 4. The spatial distributions of final slip of sixty simulation events for three values of $R$ : (a) for $10 \%\left(\gamma_{o}\right.$ min $\left.=0.90\right),(b)$ for $6 \%\left(\gamma_{o}\right.$ min $\left.=0.94\right)$, and (c) for $2 \%\left(\gamma_{o \text { min }}=0.98\right)$, when $s=4, v_{o}=1, \alpha=10^{-2}, \beta=1.5 \times 10^{-2}$, and $\Delta=10^{-3}$. The distribution of the breaking strengths is a fractal function with $D=1.5$. The symbols used are the same as those shown in Fig. 3.

the ratio $\Delta\left(=D_{c} / D_{o}\right)$ are two frictional factors in affecting the motions of sliders. As mentioned above, for most of cases $v_{o}$ is set to be 1 . The parameter $D_{c}$ is affected by several factors (cf. Marone, 1998). Its values measured from laboratory experiments are generally in the range of $10^{-6}-$ $10^{-5} \mathrm{~m}$, which are many orders of magnitude smaller than field-based estimates and those values required by theoretical simulations of ruptures (cf. Beeler and Tullis, 1996). In this study we consider four values of $D_{c}$, i.e., $10^{-4} \mathrm{~m}, 10^{-3} \mathrm{~m}$, $10^{-2} \mathrm{~m}$, and $10^{-1} \mathrm{~m}$, which are almost between the laboratory values and the field-based ones. Hence, the values of $\Delta$ related to them are $10^{-4}, 10^{-3}, 10^{-2}$, and $10^{-1}$, respectively, because $D_{o}=1 \mathrm{~m}$. The number of sliders in use is $2^{7}=128$. The time step $\delta \tau$ must be a factor in affecting numerical results. A big value of $\delta \tau$ can cause several starting points for one event and is able to produce numerical instability. However, the simulation results for various $\delta \tau$ 's are similar when $\delta \tau<0.05$. In other words, numerical stability exists when $\delta \tau<0.05$. For resulting in numerical stability, the time step size is $\delta \tau=0.01$ (or $\delta t=0.01 \mathrm{sec}$ ) in the following computations. Displayed also in the following figures are the dimensionless maximum and minimum displacements (denoted as $S_{\max }$ and $S_{\min }$, respectively).
For exploring the effects due to the distribution of the breaking strengths, we consider three kinds of spatial distributions of the breaking strengths: a fractal distribution with $D=1.5$, a random distribution, and a uniform distribution. For the first two distribution, $\gamma_{o \text { min }}=0.98$. For the last one, the breaking strength is set to be 0.98 at slider 9 and to be 1 at the rest sliders. The values of $s, v_{o}$, and $\Delta$ are 1,1 , and $10^{-3}$, respectively. The spatial distributions of final slip of sixty events for the three distributions of the breaking strengths are shown in Fig. 3. For the uniform distribution, three large events, rupturing along the whole fault (expect for the two end sliders), appear in the first three time intervals. After that, a large event, rupturing almost along the whole fault, followed by two small ones, occurring at the two second-end sliders, appears repeatedly. When the fractal and random distributions are applied, the ruptures are non-uniform along the fault. For the fractal distribution (see Fig. 3(a)), the fault can be divided mainly into two segments: one, in the righthanded side, with lower breaking strengths and the other, in the left-handed side, with higher breaking strengths. In addition, there is a transition zone, where the breaking strengths increase from right to left, between the two above-mentioned segments. A rupture cycle along the fault essentially con- 

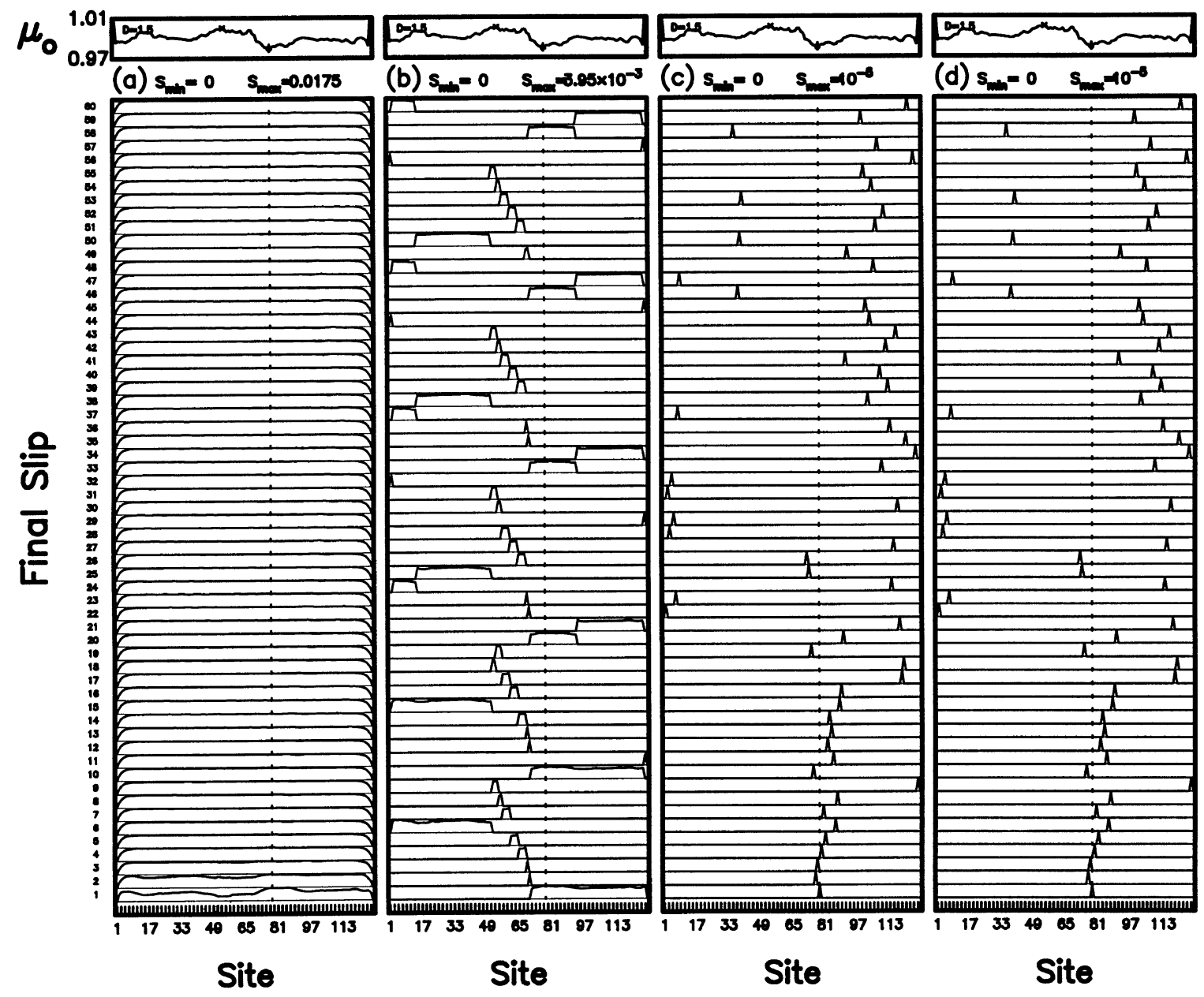

Fig. 5. The spatial distributions of final slip of sixty simulation events for two values of $\beta$ and $v_{o}$ : (a) for $\beta=10^{-2}$ and $v_{o}=1$, (b) for $\beta=2 \times 10^{-2}$ and $v_{o}=1$, (c) for $\beta=10^{-2}$ and $v_{o}=10^{-9}$, and (d) for $\beta=2 \times 10^{-3}$ and $v_{o}=10^{-9}$, when $s=4, \alpha=10^{-2}$, and $\Delta=10^{-3}$. The distribution of the breaking strengths is a fractal function with $D=1.5$ and $R=2 \%$. The symbols used are the same as those shown in Fig. 3 .

sists of three steps: First, a larger-sized event occurs along the right segment with lower breaking strengths. Secondly, few small events appear in the transition zone and at the two second-end sliders. Finally, the other larger-sized event takes place along the left segment with higher breaking strengths. The energy provided by the moving plate releases completely during a rupture cycle. Such a rupture cycle almost appears repeatedly. Of course, the number of small events, occurring in the transition zone, changes with time. However, these small events play a minor role for energy release during a rupture cycle. The number of rupture cycles for the uniform distribution is larger than that for each of the two inhomogeneous distributions. For each case, almost all the sliders, except for the two end ones, slide in a rupture cycle. The total rupture time for a rupture cycle is approximately a constant, yet about ten times smaller the time interval between two rupture cycles. This phenomenon can also be seen in the following cases with different model parameters.

Figure 3 also shows that the maximum final slip somewhat depends upon the type of distribution: the largest one for the uniform distribution, the intermediate one for the fractal distribution, and the smallest one for the random distribution. Of course, this statement is not necessary to be a general one, because different fractal dimensions and different values of $\alpha$ and $\beta$ could lead to different results. Different fractal dimensions produce different fractal distributions, thus being able to yield different spatial distributions of final slip. However, tests show that when the coefficients of friction law are fixed, the maximum final slips for different fractal distributions with fractal dimensions varying from 1.1 to 1.9 are essentially similar, and the spatial distributions of slip for different fractal dimensions are also similar to that displayed in Fig. 3. (The spatial distributions of the displacements for different fractal distributions of the breaking strengths are not shown in this study.) Hence, the above-mentioned statement can be correct when the values of $\alpha$ and $\beta$ are fixed. Results obviously show the importance of inhomogeneous breaking strengths (the highest one for the random distribution, the intermediate one for the fractal distribution and the lowest one for the uniform distribution) on the spatial distribution and the maximum value of final slip.

For an inhomogeneous distribution of the breaking strengths, in addition to the pattern of inhomogeneities, the degree of inhomogeneities would be also a significant factor in affecting slip complexity. In this study, the parameter $R$ is considered as an indication to show the degree of inho- 


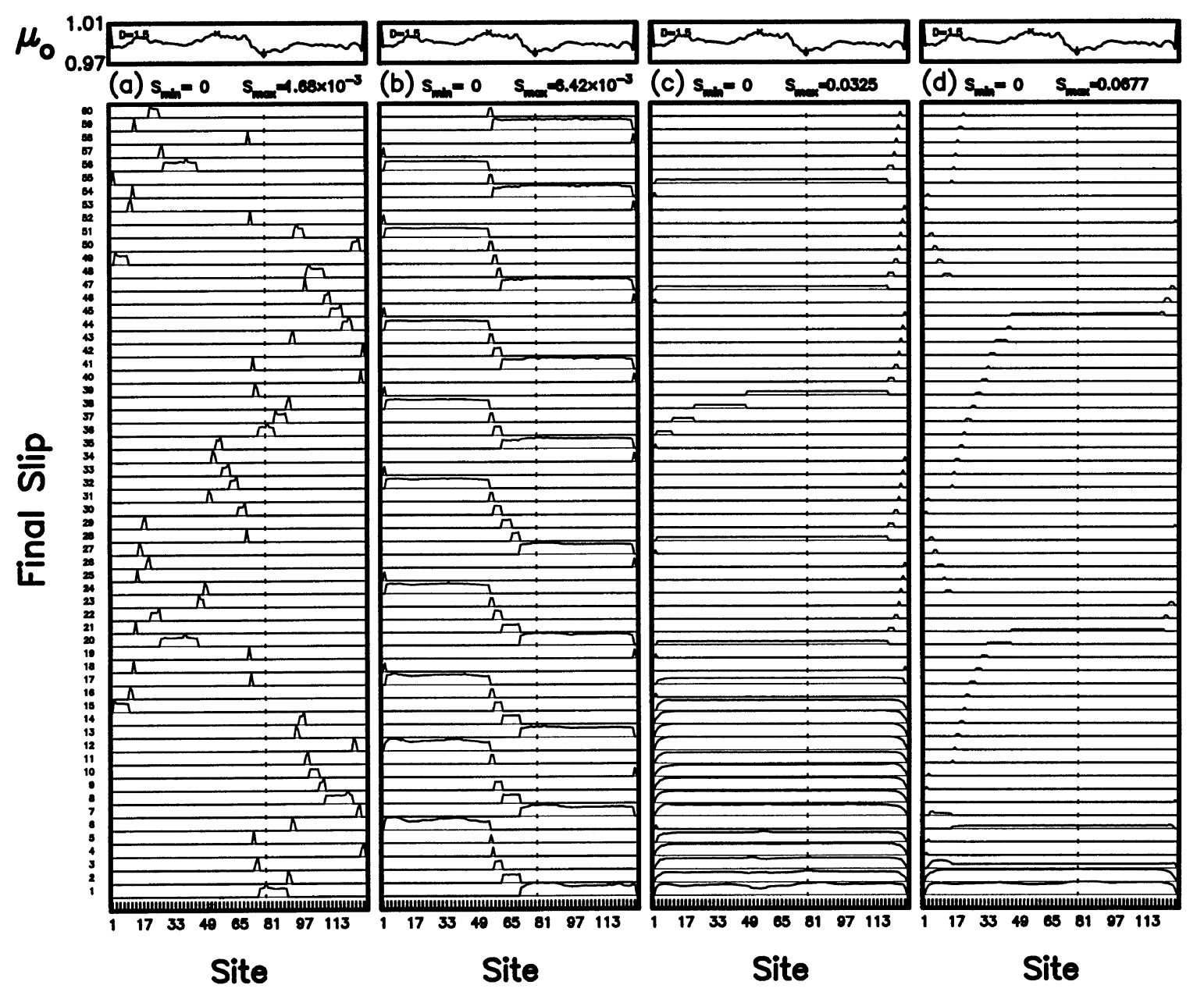

Fig. 6. The spatial distributions of final slip of sixty simulation events for four values of $\Delta$ : (a) for $10^{-4}$, (b) for $10^{-3}$, (c) for $10^{-2}$, and (d) for $10^{-1}$, when $s=4, \alpha=10^{-2}, \beta=1.5 \times 10^{-2}$, and $v_{o}=1$. The distribution of the breaking strengths is a fractal function with $D=1.5$ and $R=2 \%$. The symbols used are the same as those shown in Fig. 3.

mogeneities. The spatial distributions in final slip of sixty simulation events for three value of $R$, i.e., $10 \%, 6 \%$, and $2 \%$, which are related to $\gamma_{o \text { min }}=0.90,0.94$, and 0.98 , respectively, for a fractal distributions (with $D=1.5$ ) of the breaking strengths are shown in Fig. 4. The values of $s, v_{o}$, and $\Delta$ used are 1,1 , and $10^{-3}$, respectively. When $R=2 \%$, the simulation results (see Fig. 4(c)) are the same as those shown in Fig. 3(a), and the results were described previously. The simulation results for $R=10 \%$ (as shown in Fig. 4(a)) show that all events are small, and each of them is associated with either a small number of slid sliders or only one slid slider. The first 52 events belong to a rupture cycle. The energy in the fault system exerted by the moving plate completely releases after the occurrences of the 52 events. One could consider that the 52 events are sub-events of a large one. However, the total rupture time of the 52 events is too long to consider them to be sub-events of a large one. The simulation results for $R=6 \%$ (as shown in Fig. 4(b)) show that there are two rupture cycles, each occurring in a somewhat long time interval, and in each cycle there are small and intermediate-sized events. The seismicity pattern for $R=6 \%$ seems to be a middle state between those for $R=2 \%$ and $10 \%$. However, the values of the maximum final slip for the three values of $R$ are almost equal and independent upon $R$. For each case, except for the two end sliders, almost all sliders move in each rupture cycle. In addition, the total rupture times for individual cycles in different diagrams are approximately equal. Of course, the total times for the sixty events are different for the three cases: the largest one for $R=2 \%$, the second largest one for $R=6 \%$, and the smallest one for $R=10 \%$.

In addition to the effects due to the distribution of the breaking strengths, we need to study the frictional effects. As mentioned above, the major factors of the friction law include $\alpha, \beta$, and $\Delta$, and $v_{o}$ is set to be 1 . For comparison, we also consider two cases with $v_{o}=10^{-9}$. Figure 5 shows the plots for two values of $\beta$ and two values of $v_{o}$ : (a) for $\beta=1 \times 10^{-2}$ and $v_{o}=1$, (b) for $\beta=2 \times 10^{-2}$ and $v_{o}=1$, (c) for $\beta=1 \times 10^{-2}$ and $v_{o}=10^{-9}$, and (d) for $\beta=2 \times 10^{-3}$ and $\nu_{o}=10^{-9}$, when $s=4, \alpha=10^{-2}$, and $\Delta=10^{-3}$. The distribution of the breaking strengths is a fractal function with $D=1.5$ and $R=2 \%$. The case for $\beta=1.5 \times 10^{-2}$ and $v_{o}=1$ can be seen in Fig. 6(b). These plots for $v_{o}=1$ show that large events, rupturing almost along the whole fault, are generated when $\beta=1 \times 10^{-2}$ or $\alpha-\beta=0$, and both the size of the largest event and the 

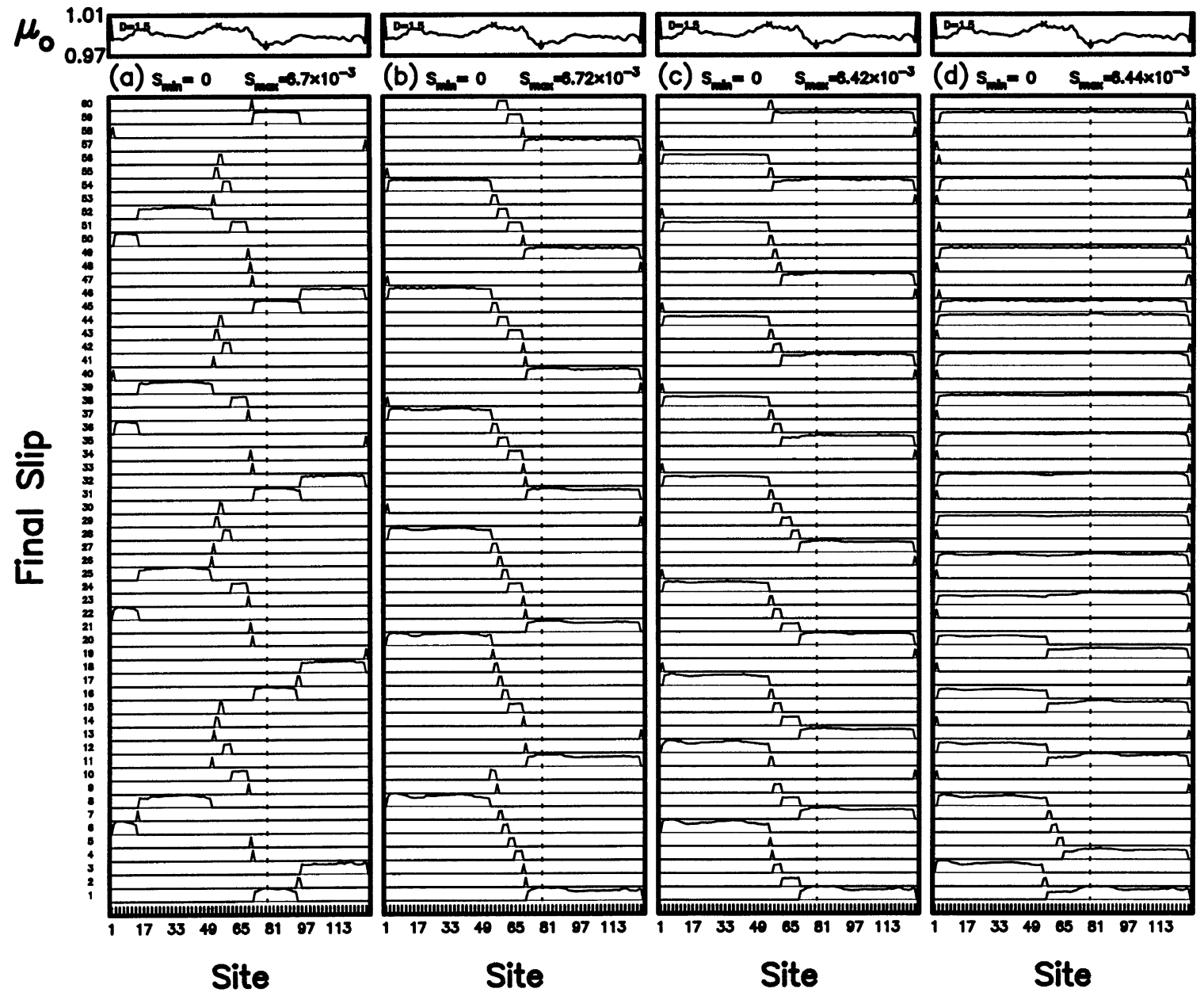

Fig. 7. The spatial distributions of final slip of sixty simulation events for four values of $s$ : (a) for 2, (b) for 3, (c) for 4 , and (d) for 5 , when $\alpha=10^{-2}$, $\beta=1.5 \times 10^{-2}, v_{o}=1$, and $\Delta=10^{-3}$. The distribution of the breaking strengths is a fractal function with $D=1.5$ and $R=2 \%$. The symbols used are the same as those shown in Fig. 3.

maximum final slip decrease with increasing $\beta$. Figures 5(c) and $5(\mathrm{~d})$ show that when $v_{o}=10^{-9}$, the seismicity patterns and the values of the maximum final slip for the two values of are the same. In the following simulations, the values of $\alpha$ and $\beta$ are $10^{-2}$ and $1.5 \times 10^{-2}$, respectively.

Figure 6 shows the spatial distributions of final slip for sixty simulation events for the four values of $\Delta$, i.e., $10^{-4}$, $10^{-3}, 10^{-2}$, and $10^{-1}$, when $s=4$ and $v_{o}=10^{-3}$. The distribution of the breaking strengths is a fractal function with $D=1.5$ and $R=2 \%$. It is evident that large $\Delta$ is more capable of generating larger-sized events than small $\Delta$, and the maximum final slip increases with $\Delta$. There are several rupture cycles for $\Delta>10^{-4}$, but only two cycles for $\Delta=10^{-4}$. The rupture times for individual cycles for the four cases are almost equal.

Finally, the effects caused by the stiffness ratio are taken into account. Figure 7 shows the spatial distributions of final slip for sixty simulation events for four values of $s$, i.e., 2 , 3 , 4, and 5 , when $v_{o}=1$ and $\Delta=10^{-3}$. The distribution of the breaking strengths is the same as that in Fig. 6. It is obvious that the range of ruptures increases slightly with $s$, but the maximum final slip does not.

\section{Discussion}

The inhomogeneous distribution of the breaking strengths and non-linearity of velocity- and state-dependent friction are usually considered as two important factors in affecting slip complexity. The debate about which is the predominant factor has been lasted for a long time. Carlson and her co-author (cf. Carlson and Langer, 1989; Carlson et al., 1991) stressed that nonlinear velocity-dependent friction is the major factor. Cochard and Madariaga $(1994,1996)$ also emphasized the importance of friction on slip complexity. On the other hand, Rice (1993) strongly argued that the complexity comes from the self-organized models and the BK models has been sensitive to the (inherent) spatial discretizations used. $\mathrm{He}$ proposed that the dynamic effect suggested by Carlson and her co-authors is not the major factor in influencing slip complexity in the earthquake faults. He also stated that models that incorporate approximately geometric fault-zone disorder can produce slip histories with features that are comparable to observations. Based on a model through the discretization from a continuum one, Shaw (1994) stressed that slip complexity is mainly caused by non-linearity of velocityand state-dependent friction rather than by the matter that the grid size $h$ is larger than a critical length $h^{*}$ of the model. 
Of course, the difference in the conclusions obtained by the rival groups might also be due to the use of different friction laws used by different authors.

Figure 3 obviously show that when the distribution of the breaking strengths is uniform, the spatial distribution of simulated final slip is also uniform whatever the values of model parameters are. Figure 4 shows the effects caused by the degree of inhomogeneity, represented by the parameter $R$, on slip complexity. A decrease in $R$ results in an increase in the capability to generate large events. This is due to an increase in the degree of homogeneity with decreasing $R$. The distribution of the breaking strengths is uniform when $R=0$. Results shown in Figs. 3 and 4 indicate that the degree and the pattern of inhomogeneous breaking strengths over the fault plane are the major factors in affecting slip complexity, while non-linearity of velocity- and state-dependent friction is less significant. However, the present results cannot directly confirm Rice's proposition. In addition, Fig. 4 also shows that the capability to generate larger-sized earthquakes decreases with increasing $R$, and only small events are produced when $R=10 \%$.

When the distribution of the breaking strengths is inhomogeneous, the effects on slip complexity caused by nonlinear velocity- and state-dependent friction obviously appear. The main parameters in the velocity- and state-dependent friction law include $\alpha$ (the parameter for the direct effect), $\beta$ (the parameter for the evolution effect), $\Delta$ (the dimensionless characteristic slip distance), and $v_{o}$ (the dimensionless reference velocity). Figure 5(a) (for $\beta=10^{-2}$ ), Fig. 5(b) (for $\beta=2 \times 10^{-2}$ ), and Fig. 6(b) (for $\beta=1.5 \times 10^{-2}$ ) show the effect of $\beta$ as well as $\alpha-\beta$ on slip complexity when $v_{o}=1$. When $\beta=10^{-2}$ or $\alpha-\beta=0$, only large events, rupturing almost along the whole fault, are generated. The size of the largest event and the maximum final slip decrease with increasing $\beta$. This indicates that when $\nu_{o}=1$, large $\beta$ is not appropriate for the generation of larger-sized events. An increase in $\beta$ (or $b$ ) results in an increase in the increasing rate of the dynamic friction strength with velocity. The effect is the same as that for decreasing $\Delta$ (or decreasing $D_{c}$ ) as shown in Fig. 2. In addition, Figs. 5(c) and 5(d) also show that no larger-sized events can be yielded when $v_{o}=10^{-9}$. As shown in Fig. 2(a) for $v_{o}=10^{-9}$, the dynamic friction strength obviously increases with velocity. Hence, after a slider moves, the increasing dynamic friction force resists the slider to slide further. Hence, small $v_{o}$ cannot result in large events.

Figure 6 shows the spatial distributions of final slip for sixty simulation events for the four values of $\Delta$, i.e., $10^{-4}$, $10^{-3}, 10^{-2}$, and $10^{-1}$, when $s=4$ and $v_{o}=10^{-3}$. It is evident that large $\Delta$ is more capable of generating larger-sized events than small $\Delta$, and the maximum final slip increases with $\Delta$. From Fig. 2(e) with $v_{o}=10^{-3} \mathrm{~m} / \mathrm{s}\left(\right.$ or $v_{o}=10^{-3}$ ), we can see that the friction strength and the increasing rate of friction strength at low velocities generally increases with decreasing $D_{c}$. This indicates that smaller $D_{c}$ (or $\Delta$ ) is less appropriate for generating larger-sized model events. This leads to the phenomena observed in Fig. 6.

The stiffness ratio, $s$, would be a factor in influencing slip complexity. The results shown in Fig. 7 display that larger $s$ is more appropriate for producing larger-sized events than smaller $s$. Wang (1995) also stated that large $s$ is more capable of generating larger-sized events, thus leading to a smaller $b$-value, than small $s$. The reasons are described as below: The coil spring (with a stiffness of $K_{c}$ ) between two sliders only transfers the mechanical energy from one slider to the nearest ones. Thus, it does not take the elastic energy in the system away. On the other hand, the leaf spring (with a stiffness $K_{l}$ ) between a slider and the moving plate plays two roles: One is to transfer the mechanical energy, exerted from the moving plate, into the system through the $K_{l} V_{p} t$ term, and the other to remove the energy through the $-K_{l} x_{i}$ term. Hence, the stiffness ratio is also a parameter representing the level of conservation of energy in the system. Larger $s$ indicates a higher level of conservation or a lower level of dissipation of energy in the system. Hence, an increase in the capability of yielding larger-sized events with $s$ must be reasonable.

Simulation results in Figs. 3-7 also show that the maximum final slip depends upon mainly the characteristic slip distance and the reference velocity, but only slightly affected by stiffness ratio, the pattern of the inhomogeneous distribution of the breaking strengths, and the degree of inhomogeneities. The former two parameters directly affect the dynamical rupture processes (see Fig. 2), but the latter three parameters do not. Hence, the former two parameters are more important on the size of the final slip than the latter three.

\section{Conclusions}

The characteristic slip, $D_{c}$, and the reference velocity, $v_{o}$, of the slip law are two important parameters affecting dynamic friction strength. The velocity-weakening process is produced when the reference velocity, $v_{o}$, is smaller than a critical value, $v_{o c}\left(=10^{-5} \mathrm{~m} / \mathrm{s}\right.$ in this study $)$, while the velocity-hardening process is yielded when $v_{o}>v_{o c}$. The changing rate of $\mu$ with sliding velocity depends on $D_{c}$.

Since the friction law itself could affect simulation results, the following conclusions mainly hold for the velocity- and state-dependent friction law used in this study. Simulation results show that slip complexity depends on the heterogeneity of the breaking strengths and nonlinear velocity- and statedependent friction. The former is more important than the latter. When the distribution of the breaking strengths is homogeneous, only large events, which rupture almost uniformly along the fault, are generated. When the distribution of the breaking strengths is inhomogeneous, the friction law and the stiffness ratio are also significant factors in affecting slip complexity.

Acknowledgments. The authors would like to express thanks to two anonymous reviewers for helpful comments and suggestions. This study was financially supported from Academia Sinica and the National Sciences Council, ROC under Grant NSC87-2116-M001-020.

\section{References}

Beeler, N. M. and T. E. Tullis, Self-healing slip pulses in dynamic rupture models due to velocity-dependent strength, Bull. Seism. Soc. Am., 86 1130-1148, 1996

Burridge, R. and L. Knopoff, Model and theoretical seismicity, Bull. Seism. Soc. Am., 57, 341-371, 1967.

Carlson, J. M., Time intervals between characteristic earthquakes and cor- 
relation with smaller events: An analysis based on a mechanical model of fault, J. Geophys. Res., 96, 4255-4267, 1991.

Carlson, J. and J. S. Langer, Mechanical model of an earthquake fault, Phys. Rev. A, 40, 6470-6484, 1989.

Carlson, J. M., J. S. Langer, B. E. Shaw, and C. Tang, Intrinsic properties of a Burridge-Knopoff model of an earthquake fault, Phys. Rev. A, 44, 884-897, 1991.

Cochard, A. and R. Madariaga, Dynamic faulting under rate-dependent friction, Pure Appl. Geophys., 142, 419-445, 1994.

Cochard, A. and R. Madariaga, Complexity of seismicity due to highly rate-dependent friction, J. Geophys. Res., 101, 25,321-25,336, 1996.

Das, S. and K. Aki, Fault planes with barriers: a versatile earthquake model, J. Geophys. Res., 82, 5658-5670, 1977.

Dieterich, J. H., Modeling of rock friction 1. Experimental results and constitutive equations, J. Geophys. Res., 84, 2161-2168, 1979.

Kanamori, H., Mechanics of Earthquake, Annu. Rev. Earth Planet. Sci., 22, 207-237, 1994.

Kanamori, H. and G. Stewart, Seismological aspects of the Guatemala earthquake of February 4, 1976, J. Geophys. Res., 83, 3427-3434, 1978.

Knopoff, L., The organization of seismicity on fault networks, Proc. Natl. Acad. Sci., USA, 93, 3830-3837, 1996.

Marone, C., Laboratory-derived friction laws and their application to seismic faulting, Annu. Rev. Earth Planet. Sci., 26, 643-696, 1998.
Press, W. H., B. P. Flannery, S. A. Teukolsky, and W. T. Vetterling, Numerical Recipes, 818 pp., Cambridge Univ. Press, Cambridge, 1986.

Rice, J. R., Fault stress states, pore pressure distributions, and the weakness of the San Andreas Fault, in Fault Mechanics and Transport Properties of Rocks, pp. 475-503, Academic Press, 1992.

Rice, J. R., Spatio-temporal complexity of slip on a fault, J. Geophys. Res., 98, 9885-9907, 1993.

Ruina, A. L., Slip instability and state variable friction laws, J. Geophys. Res., 88, 10,359-10,370, 1983.

Saupe, D., Algorithms for random fractals, Chapter 2, in The Science of Fractal Images, edited by H. O. Peitgen and D. Saupe, pp. 71-136, Springer Verlag, New York, 1988.

Scholz, C. H., The Mechanics of Earthquakes and Faulting, 439 pp., Cambridge Univ. Press, Cambridge, 1990.

Shaw, B. E., Complexity in a spatially uniform continuum fault model, Geophys. Res. Lett., 21, 1983-1986, 1994.

Wang, J. H., Effect of seismic coupling on the scaling of seismicity, Geophys. J. Int., 121, 475-488, 1995.

Wang, J. H., A dynamical study of two one-state-variable, rate- and statedependent friction laws, Bull. Seism. Soc. Am., 1999 (in revision).

J.-H. Wang (e-mail: jhwang@earth.sinica.edu.tw) and R.-D. Hwang 